\title{
Expresión de CD44v6 en adenocarcinomas de pulmón. Asociación con altas concentraciones citosólicas de CA125
}

\section{Sr. Director:}

El CD44 es una familia de moléculas involucradas en las interrelaciones célula-célula y célula-matriz extracelular. Existe una forma estándar (CD44s) y otras variantes (CD44v) consecuencia de modificaciones post-translacionales y splicing alternativos, muchas de las cuales se consideran marcadores de metástasis e indicadores pronósticos en determinados tumores. En el campo oncológico, el interés clínico de la isoforma variante v6 (VD44v6) varía según cual sea el tumor estudiado. En el aparato respiratorio se puede constatar expresión del CD44v6 en el epitelio pulmonar normal (neumocitos tipo II preferentemente) y aquella parece jugar un papel importante en el mantenimiento de la arquitectura tisular'. Asimismo, su positividad inmunohistoquímica es más frecuente en los tumores escamosos y bronquioalveolares ${ }^{2}$. Nosotros, en este trabajo hemos querido estudiar la expresión inmunológica del CD44v6 en los adenocarcinomas y correlacionarla con otros parámetros clínico-biológicos que ayudaran a comprender su interés fisiopatológico y posible utilidad práctica. El grupo estudio incluyó 59 muestras tisulares tumorales de otros tantos pacientes afectos de adenocarcinomas de pulmón, sin tratamiento previo, de edades comprendidas entre 33 y 79 años (mediana 64), de los cuales 33 eran varones. Asimismo, en 16 casos obtuvimos, además, una muestra de tejido pulmonar aparentemente normal. El CD44v6 fue determinado mediante un EIA (Bender Diagnostics. Austria), cuyo dintel de positividad fue establecido en 5 $\mathrm{ng} / \mathrm{mg}$ prot. ${ }^{3}$. Otros parámetros analizados en las membranas celulares fueron las concentraciones de la proteína oncogénica erbB2/neu, receptor del factor de crecimiento epidérmico (EGFR) y las de CD44v5. También se analizaron las concentraciones citosólicas de CA125, pS2, activador del plasminógeno tipo tisular (t-PA), enolasa específica neuronal (NSE), cyfra 21.1 y fracción libre de la subunidad beta de la hormona gonadotrófica coriónica (HCG beta) Los valores de los parámetros biológicos fueron expresados por $\mathrm{mg}$ de proteína determinada por el método de Bradford. También se ha tenido presente el estadio clínico, grado histológico $(\mathrm{GH})$, ploidía y fase $S(\mathrm{FS})$, determinadas estas dos últimas mediante citometría de flujo ( Becton Dickinson. Fascam. EEUU) en muestras en fresco. Como las concentraciones de los parámetros biológicos no siguieron una distribución gaussiana, hemos empleado el test de MannWhitney, así como el chi cuadrado (x2), con la correción de Yates cuando fue necesaria, para la comparación de variables cualitativas. Los resultados han sido expresados mediante la mediana y el intervalo. Dadas las bajas concentraciones de pS2, HCGbeta and t-AP en estos tumores, hemos utilizado los porcentajes de positividades, tomando como dinteles $1,0,02$ y $1 \mathrm{ng} / \mathrm{mg}$ prot. respectivamente. Una diferencia se consideró estadística cuando el valor de $p$ fue inferior a 0,05 .

En los adenocarcinomas de pulmón, la positividad para el CD44v6 fue apreciada en 34 de los 59 casos $(57.6 \%)$ y casi difirió estadísticamente (p: 0.083) de la constatada en los tejidos pulmonares normales de los mismos pacientes $(13 / 16 ; 81.2 \%)$. Los tumores CD44v6 positivos presentaron exclusivamente, tal como se expone en la Tabla I, mayores concentraciones de CA1 25 (p: 0.008) y fueron más frecuentemente CD44v5 positivos $(p<0.001)$. La utilidad clínica de la expresión de CD44v6 varía según el tumor que consideremos, pudiendo comportarse como un factor de buen o mal pronóstico. Menos trabajos existen en relación con su comportamiento en los carcinoma pulmonares no microcíticos y por ello hemos querido estudiar, mediante un método inmunoenzimático, su concentración en las membranas celulares de adenocarcinomas pulmonares. Hemos observado expresión de la isoforma en el $57,6 \%$ de los casos, siendo menor a la observada en los tejidos pulmonares normales de los mismos. Este mismo comportamiento del CD44v6 entre tejido tumoral y normal ha sido descrito recientemente $^{4}$, apoyando su posible papel en la transformación tumoral. Sabemos que la isoforma CD44v6 es expresada por el epitelio pulmonar normal donde juega un importante papel en el mantenimiento de la estructura celular y que, asimismo, es un indicador de la diferenciación escamosa, si bien es posible detectarla también en los adenocarcinomas, aunque de un modo menos frecuente. En los carcinomas no microcíticos la positividad para esta isoforma parece ser indicadora 
Distribución de los valores de los diferentes parámetros clínico-biológicos en los adenocacinomas de pulmón clasificados en función de la positividad para el CD44v6 (> $5 \mathrm{ng} / \mathrm{mg}$ prot.)

\begin{tabular}{|c|c|c|c|c|c|c|c|}
\hline \multirow{2}{*}{ Parámetro } & \multicolumn{3}{|c|}{$>5(34$ casos $)$} & \multicolumn{3}{|c|}{$</=5$ (25 casos) } & \multirow[b]{2}{*}{$\mathbf{p}$} \\
\hline & Intervalo & & Mediana & Intervalo & & Mediana & \\
\hline ErbB2* & $656-20590$ & & 3080 & $171-117750$ & & 3420 & ns \\
\hline CA $125^{* *}$ & $1-9225$ & & 109,5 & $1-616$ & & 37,8 & 0,008 \\
\hline Tamaño+ & $1,5-10$ & & 4,0 & $2-10$ & & 4,5 & ns \\
\hline $\mathrm{NSE}^{* * *}$ & $26,8-878$ & & 269 & $56,7-1004$ & & 275 & ns \\
\hline Cyfra $21.1^{* * *}$ & $59,6-6774$ & & 1163 & $24,8-6194$ & & 1092 & ns \\
\hline EGFR & $3,1-204$ & & 22 & $1-368$ & & 18,8 & ns \\
\hline GH3 & & $16 / 34$ & & & $10 / 25$ & & ns \\
\hline Estadio I & & $22 / 34$ & & & $10 / 25$ & & ns \\
\hline Estadio II & & $3 / 34$ & & & $5 / 25$ & & ns \\
\hline Estadio III & & $9 / 34$ & & & $10 / 25$ & & ns \\
\hline $\mathrm{FS}>12,2 \%++$ & & $14 / 26$ & & & $8 / 18$ & & ns \\
\hline $\mathrm{FS}>20,8+++$ & & $7 / 26$ & & & $4 / 18$ & & ns \\
\hline $\mathrm{pS} 2>1^{* * *}$ & & $11 / 34$ & & & $7 / 25$ & & ns \\
\hline Aneuploides & & $25 / 29$ & & & $17 / 19$ & & ns \\
\hline $\mathrm{CD} 44 \mathrm{v} 5+{ }^{* * *} /{ }^{*}$ & & $22 / 34$ & & & $5 / 25$ & & $<0,001$ \\
\hline$t-A P>1^{* * *}$ & & $21 / 34$ & & & $15 / 25$ & & ns \\
\hline HCGbeta $>0.02^{* * *}$ & & $19 / 34$ & & & $13 / 25$ & & ns \\
\hline
\end{tabular}

$\mathrm{NHI} / \mathrm{mg}$ prot.*

$\mathrm{U} / \mathrm{mg}$ prot. ${ }^{* *}$

$\mathrm{ng} / \mathrm{mg}$ prot. ${ }^{* * *}$

$+: \mathrm{cm}$

++: mediana de todo el grupo

+++ : percentil 75 de todo el grupo

${ }^{* * *} /{ }^{*}:>3 \mathrm{ng} / \mathrm{mg}$ prot. (dintel de positividad)

NSE: enolasa específica neuronal

EGFR: receptor del factor de crecimiento epidérmico

FS: fase de síntesis celular

t-AP: activador del plasminógeno tipo tisular

HCGbeta: fracción libre de la subunidad beta de la hormona gonadotrófica coriónica

$\mathrm{GH}$ : grado histológico

de un peor comportamiento y evolución. A este respecto, Hirata y cols. ${ }^{5}$ demuestran que la expresión de CD44v6 se asocia en los carcinomas no microcíticos estadio I con un peor pronóstico y evolución, correlacionándose con una mayor proliferación celular, reflejada mediante la tinción inmunohistoquímica del antígeno nuclear de proliferación celular (PCNA) y comportándose como un factor pronóstico independiente tras análisis multivariante. Sin embargo, otros grupos han constatado hechos diferentes ${ }^{6-8}$. En nuestra experiencia, la expresión de CD44v6 en los adenocarcinomas se asoció a mayores concentraciones de CA125 y la positividad para la isoforma variante CD44v5. En relación con el CA 125 sabemos que sus concentraciones séricas se correlacionan, en los tumores no microcíticos, con el estadio clínico y la efectividad de la te- rapia, pudiendo ser un factor pronóstico independiente en los estadios avanzados, 10; asimismo, su concentración citosólica también se asocia con una menor supervivencia en esos subtipos histológicos ${ }^{11,12}$. Existen pocas referencias respecto al comportamiento del CD44v5 en los adenocarcinomas pulmonares. Nosotros (manuscrito en preparación), hemos observado que su positividad se constata en el $49,1 \%$ de los adenocarcinomas y que ello se acompaña de mayores concentraciones de CA125 que rozan la significación estadística ( $p: 0,063)$.

Los resultados anteriores nos inducen a las siguientes consideraciones: 1) es posible detectar la expresión de CD44v6 en un alto porcentaje de los adenocarcinomas de pulmón; 2) la positividad para el CD44v6 no se correlacionó con ninguno de los parámetros clínicos 


\section{A. Ruibal y cols.}

habituales, pero sí con mayores concentraciones de CA 125 y una superior positividad para el CD44v5, lo cual apoyaría que fuese reflejo de un peor comportamiento-evolución, si bien son necesarios otros estudios para poder precisar el valor clínico de la expresión de esta molécula de adhesión en este subtipo de tumores pulmonares.

\section{A. Ruibal*, J. Barandela*, I. Núñez*, J. Rodríguez** \\ * Servicio de Medicina Nuclear Hospital Clínico Universitario Complejo Hospitalario Universitario Santiago de Compostela \\ ** Servicio de Cirugía Torácica Hospital Central de Asturias. Oviedo}

\section{Bibliografía}

1. Kasper M, Gunther U, Dall P, Kayser K, Schuh D, Haroske G, Muller M. Distinct expression patterns of CD44 isoforms during human lung development and pulmonary fibrosis. Am J Respir Cell Moll Biol 1995; 13:64856.

2. Fasano M, Sabatini MT, Wieczorek R, Sidhu G, Goswami S, Jagirdar J. CD44 and its v6 spliced variant in lung tumors: a role in histogenesis? Cancer 1997; 80:34-41.

3. Ruibal A, Schneider J, del Río MC, Arias JI, Núñez MI, Tejerina A. La expresión de la molécula de adhesión CD44v6 en carcinomas ductales infiltrantes de mama se asocia con la hormonodependencia. Nuestra experiencia con 168 casos. Rev Esp Med Nuclear 2000; 19:350-5.

4. Shimbori M, Kijama H, Sato S, Yoshida H, Sato T, Terasaki-Fukuzawa $Y$, et al. Expressión of CD44 in primary lung carcinomas using histological and cytological analyses. Anticancer Res 2003; 23:115-121.

5. Hirata T, Fukuse T, Naiki H, Hitomi S, Wada H. Expression of CD44 variant exon 6 in stage I non small cell lung carcinoma as a prognostic factor. Cancer Res 1998; 58:1 108-10.

6. Suzuki H, Yamashiro K. Reduced expression of CD44v3 and $\mathrm{v} 6$ is related to invasion in lung adenocarcinoma. Lung Cancer 2002; 38:137-41.

7. Ramasami S, Kerr KM, Chapman AD, King G, Cockburn $J S$, Jeffrey RR. Expression of CD44v6 but not E-cadherin or beta catenin influences prognosis in primary pulmonary adenocarcinoma. J Pathol 2000; 192:427-32.

8. Pirinen R, Hirvikoski P, Bohn J, Kellokoski J, Moisio K, Viren $M$, et al. Reduced expression of CD44v3 variant isoform is associated with unfavourable outcome in non small cell lung carcinoma. Human Pathol 2000; 31: 1088-05.

9. Salgia R, Harpole D, Herndon JE 2nd, Pisick E, Elias A, Skarin AT. Role of serum tumor markers CA1 25 and CEA in non-small cell lung cancer. Anticancer Res 2001; 21:1241-6.

10. Díez M, Pollan M, Maestro ML, Ortega MD, Gómez A, Sánchez Pernaute $A$, et al. Concordance between serum and cytosolic levels of CEA, CA125 and SCC antigens in patients with non-small cell lung cancer. Anticancer Res 1995; 15:2811-7.

11. Picardo AL, Díez M, Torres A, Maestro ML, Ortega D, Hernando $F$, et al. Analysis of the prognostic significance of cytosolic determination of CA 125 tumor-associated antigen, carcinoembryonic antigen and squamous cell carcinoma antigen in patients with non-small cell lung carcinoma. Cancer 1996; 77:1066-72.

12. Díez M, Torres A, Maestro ML, Ortega MD, Gómez A, Pollan $M$, et al. Prediction of survival and recurrence by serum and cytosolic levels of CEA, CA125 and SCC antigens in resectable non-smal cell lung cancer. $\mathrm{Br} \mathrm{J}$ Cancer 1996; 73:1248-54.
Correspondencia:

Dr. A. Ruibal Morell

Servicio de Medicina Nuclear

Hospital Clínico Universitario

Complejo Hospitalario Universitario Bloque D

E-15706 Santiago de Compostela 